\title{
EDITORIAL
}

\section{Risks, returns and reassurance}

\author{
The ongoing debate on the creation of an abbreviated regulatory pathway for 'follow-on' \\ versions of innovator biologics in the United States highlights questions about the current \\ balance between incentives for innovation and price competition in general.
}

Almost a quarter of a century ago, the US Congress passed landmark amendments to the core legislation on the regulation of pharmaceuticals. Known as the Hatch-Waxman Act, the changes had two main aspects ${ }^{1}$. The first was restoration of a period of patent protection for new drugs to compensate for the time taken for their clinical development and regulatory review. The second was creation of a mechanism for abbreviated approval of generic versions - based on the data supporting the original drug's safety and efficacy - following patent expiry or invalidation. This second aspect of the Act has created a highly successful generic drugs industry, saving health-care payers considerable costs.

However, at present, for a major class of therapeutics - biologics such as monoclonal antibodies - an abbreviated regulatory pathway analogous to that for generic small-molecule drugs created by the Hatch-Waxman Act does not exist in the United States. Unsurprisingly, given the higher costs associated with several successful biologics, and the rising health-care expenditure on them, creating such a pathway for 'follow-on' biologics has been a focus of recent debate.

A core issue of the debate is the same as one faced at the time the Hatch-Waxman Act was passed: balancing incentives for the development of innovative drugs with stimulation of price competition. However, differences in the characteristics of biologics compared with smallmolecule drugs present new challenges to achieving the desired balance.

One key difference is the extent to which the patents for a biologic drug provide protection for the innovator company against potential competitors. Compared with typical patents for small-molecule drugs, it seems that the patents for biologics could be considerably more vulnerable to challenges or circumvention. For this reason, data exclusivity - a complementary form of intellectual-property protection - has been prominent in the follow-on biologics debate. In particular, opinions have differed widely on the extent of the period of data exclusivity before a company could make an abbreviated regulatory filing for a follow-on biologic that used the innovator company's data on the original biologic's safety and efficacy. For example, one legislative proposal has proposed no data exclusivity, while another has a 14-year provision.
As highlighted by Grabowski ${ }^{2}$ in our June issue, data exclusivity could be crucial in providing innovator companies with the opportunity to recoup their investment before follow-on competitors can enter the market. In this analysis, the 'break even' point for a typical new biologic was $\sim 13-16$ years after launch ${ }^{2}$. So, without the reassurance of a substantial period of data exclusivity, the incentive to invest in the long and risky process of developing a novel biologic could be considerably reduced. This might have a particularly chilling effect on the funding sources for smaller companies, which have become an increasingly important source of innovation.

However, it is not only the balance between the return on investment for innovative biologic developers and price competition that might be well addressed through data exclusivity. For example, as recognized in some legislative proposals, investment in discovering new indications for existing drugs or studying them in under-served populations could also be stimulated by providing additional data exclusivity if successful. Other key issues that require further time and investment postapproval to be addressed optimally — such as providing data on the cost-effectiveness of novel drugs - might be tackled using a similar strategy.

The potential for data exclusivity to provide greater reassurance for companies about the minimum period before follow-on competitors could emerge than is provided by patents could also be relevant for smallmolecule drugs. Indeed, it is now common for patents for highly successful small-molecule drugs to come under threat early in the drug's life cycle, in part owing to incentives to challenge them created by the HatchWaxman Act. In this respect, it is noteworthy that the European Union has recently harmonized a 10-year data exclusivity period for both new small-molecule drugs and new biologics, as well as providing an additional year if significant new indications are identified ${ }^{2}$. So, as the debate on follow-on biologics continues in the US this year, it seems that there could also be merit in re-evaluating the existing balances between innovation and competition for small-molecule drugs originally laid down by the Hatch-Waxman Act.

Mossinghoff, G. J. Food Drug Law J. 54, 187-194 (1999). 2. Grabowski, H. Nature Rev. Drug Discov. 7, 479-488 (2008). 